\title{
TeV Gamma-Ray Absorption and the Intergalactic Infrared Background
}

\author{
F. W. Stecker \\ Laboratory for High Energy Astrophysics, NASA Goddard Space Flight \\ Center, Greenbelt, MD 20771, USA
}

\begin{abstract}
In this paper, I will take a synoptic approach to determining the intergalactic infrared radiation field (IIRF). This approach draws on both the multi-TeV $\gamma$-ray observations and the infrared background observations and relates them via the semi-empirical modelling method of Malkan \& Stecker. I discuss the evidence for an intergalactic infrared background obtained by an analysis of the HEGRA observations of the high energy $\gamma$-ray spectrum of Mrk 501 and from constraints from Mrk 421 deduced from the Whipple air Cherenkov telescope results. I will show that this evidence is in accord with the predictions made by Malkan \& Stecker (1998) for the intergalactic infrared spectral energy distribution produced by galaxies. The Malkan-Stecker predictions are also in excellent agreement with mid-infrared galaxy counts. However, there may be potential problems relating these predictions with the results of the analysis of COBE-DIRBE far infrared data. The $\gamma$-ray and COBE-DIRBE observations may also need to be reconciled. I will discuss possible ways to resolve this situation including a partial nullification of the $\gamma$-ray absorption process which can hypothetically occur if Lorentz invariance is broken.
\end{abstract}

\section{Introduction}

Shortly after the discovery that blazars can be strong sources of high energy $\gamma$ rays, we suggested that very high energy $\gamma$-ray beams from blazars can be used to measure the intergalactic infrared radiation field, since pair-production interactions of $\gamma$-rays with intergalactic IR photons will attenuate the high-energy ends of blazar spectra (Stecker, De Jager \& Salamon 1992). In recent years, this concept has been used successfully to place upper limits on the intergalactic IR field (IIRF) (Stecker \& De Jager 1993; Dwek \& Slavin 1994; Stecker \& De Jager 1997; Stanev \& Franceschini 1997; Biller et al. 1998). Determining the IIRF, in turn, allows us to model the evolution of the galaxies which produce it. As energy thresholds are lowered in both existing and planned ground-based air Cherenkov light detectors, cutoffs in the $\gamma$-ray spectra of more distant blazars are expected, owing to extinction by the IIRF. These can be used to explore the redshift dependence of the IIRF.

On the other hand, by modelling the intergalactic infrared and optical radiation fields as a function of redshift, one can calculate the expected $\gamma$-ray opacity 
as a function of redshift. Comparison of predicted absorption with the data can yield important new information about the evolution of the IIRF (Stecker \& De Jager 1998; Salamon \& Stecker 1998).

Exploring the IIRF with high energy $\gamma$-rays has both advantages and disadvantages over direct infrared observations. An important advantage is that since the absorption process only acts effectively in intergalactic space over large distances, the $\gamma$-ray approach does not suffer from problems of subtraction of foreground emission from both zodiacal light and interstellar dust in the Galaxy. As we have seen at this meeting, these can be formidable sources of uncertainty in determining the IIRF. Also, high energy $\gamma$-ray observations of blazar and $\gamma$ ray burst spectra at high redshifts can be used to probe the past evolution of the IIRF whereas direct infrared observations can only tell us about the present IIRF.

On the other hand, the disadvantage of the $\gamma$-ray approach is the uncertainty in the unabsorbed spectrum of the source. This uncertainty is somewhat ameliorated by the strong energy dependence of the absorption effect itself. Therefore, it is my contention that using both direct infrared and high energy $\gamma$-ray observations in a "synoptic" approach can yield the most information about the IIRF.

\section{The Opacity of Intergalactic Space Owing to the IIRF}

The formulae relevant to absorption calculations involving pair-production are given and discussed in Stecker, De Jager \& Salamon (1992), where we derived the absorption formulae with cosmological and redshift effects included. For $\gamma$-rays in the $\mathrm{TeV}$ energy range, the pair-production cross section is maximized when the soft photon energy is in the infrared range:

$$
\lambda\left(E_{\gamma}\right) \simeq \lambda_{e} \frac{E_{\gamma}}{4 m_{e} c^{2}}=1.24 E_{\gamma, T e V} \mu m
$$

where $\lambda_{e}=h /\left(m_{e} c\right)$ is the Compton wavelength of the electron. For $15 \mathrm{TeV}$ $\gamma$-rays, absorption will occur primarily by interactions with mid-infrared photons having a wavelength $\sim 20 \mu \mathrm{m}$. (Pair-production interactions actually take place with photons over a range of wavelengths around the optimal value as determined by the energy dependence of the cross section; see eq. (11)). If the emission spectrum of an extragalactic source extends beyond $20 \mathrm{TeV}$, then the extragalactic infrared field should cut off the observed spectrum between $\sim 20$ $\mathrm{GeV}$ and $\sim 20 \mathrm{TeV}$, depending on the redshift of the source (Stecker \& de Jager 1998; Salamon \& Stecker 1998).

\section{Absorption of Gamma-Rays at Low Redshifts}

Stecker \& De Jager (1998) (hereafter SD98) have calculated the absorption coefficient of intergalactic space using a new, empirically based calculation of the spectral energy distribution (SED) of intergalactic low energy photons by Malkan \& Stecker (1998) (hereafter MS98) obtained by integrating luminosity dependent infrared spectra of galaxies over their luminosity and redshift distributions. After giving their results on the $\gamma$-ray optical depth as a function of energy and 
redshift out to a redshift of 0.3, Stecker \& De Jager (1998) (SD98) applied their calculations by comparing their results with the spectral data on Mrk 421 (McEnery et al. 1997) and spectral data on Mrk 501 (Aharonian, et al. 1997).

SD98 made the reasonable simplifying assumption that the IIRF is basically in place at a redshifts $<0.3$, having been produced primarily at higher redshifts (Madau 1995; Salamon \& Stecker 1998). Therefore SD98 limited their calculations to $z<0.3$.

For the IIRF spectral energy distribution (SED), SD98 assumed two of the SEDs given in MS98 (shown in Figure 1). The lower curve in Figure 1 (adapted from MS98) assumes luminosity evolution proportional to $(1+z)^{3.1}$ out to $z=1$, whereas the middle curve assumes such evolution out to $z=2$. Evolution in stellar emissivity is expected to level off at redshifts greater than $\sim 1.5$ (Steidel 1999; Hopkins, Connolly \& Szalay 2000) Using these two SEDs for the IIRF, SD98 obtained parametric expressions for the optical depth $\tau\left(E_{\gamma}, z\right)$ for $z<0.3$, taking a Hubble constant of $\mathrm{H}_{o}=65 \mathrm{~km} \mathrm{~s}^{-1} \mathrm{Mpc}^{-1}$ (Gratton 1997).

The double-peaked form of the SED of the IIRF requires a third order polynomial to approximate the opacity $\tau$ in a parametric form. SD98 give the following approximation:

$$
\log _{10}\left[\tau\left(E_{\mathrm{TeV}}, z\right)\right] \simeq \sum_{i=0}^{3} a_{i}(z)\left(\log _{10} E_{\mathrm{TeV}}\right)^{i} \text { for } 1.0<E_{\mathrm{TeV}}<50,
$$

where the z-dependent coefficients are given by

$$
a_{i}(z)=\sum_{j=0}^{2} a_{i j}\left(\log _{10} z\right)^{j} .
$$

Table 1 gives the numerical values for $a_{i j}$, with $i=0,1,2,3$, and $j=0,1,2$. The numbers before the brackets are obtained using the lower IIRF SED shown in Figure 1; the numbers in the brackets are obtained using the middle IIRF SED. Equation (2) approximates $\tau(E, z)$ to within $10 \%$ for all values of $\mathrm{z}$ and $\mathrm{E}$ considered. Figure 2 shows the results obtained by SD98 for $\tau\left(E_{\gamma}, z\right)$.

Table 1: Polynomial coefficients $a_{i j}$

\begin{tabular}{|r|r|r|r|r|}
\hline$j$ & $a_{0 j}$ & $a_{1 j}$ & $a_{2 j}$ & $a_{3 j}$ \\
\hline 0 & $1.11(1.46)$ & $-0.26(0.10)$ & $1.17(0.42)$ & $-0.24(0.07)$ \\
1 & $1.15(1.46)$ & $-1.24(-1.03)$ & $2.28(1.66)$ & $-0.88(-0.56)$ \\
2 & $0.00(0.15)$ & $-0.41(-0.35)$ & $0.78(0.58)$ & $-0.31(-0.20)$ \\
\hline
\end{tabular}

The advantage of using empirical data to construct the SED of the IIRF, as done in MS98, is particularly indicated in the mid-infrared range. In this region of the spectrum, galaxy observations indicate more flux from warm dust in galaxies than that taken account of in more theoretically oriented models (e.g., MacMinn \& Primack 1996). As a consequence, the mid-infrared "valley" 


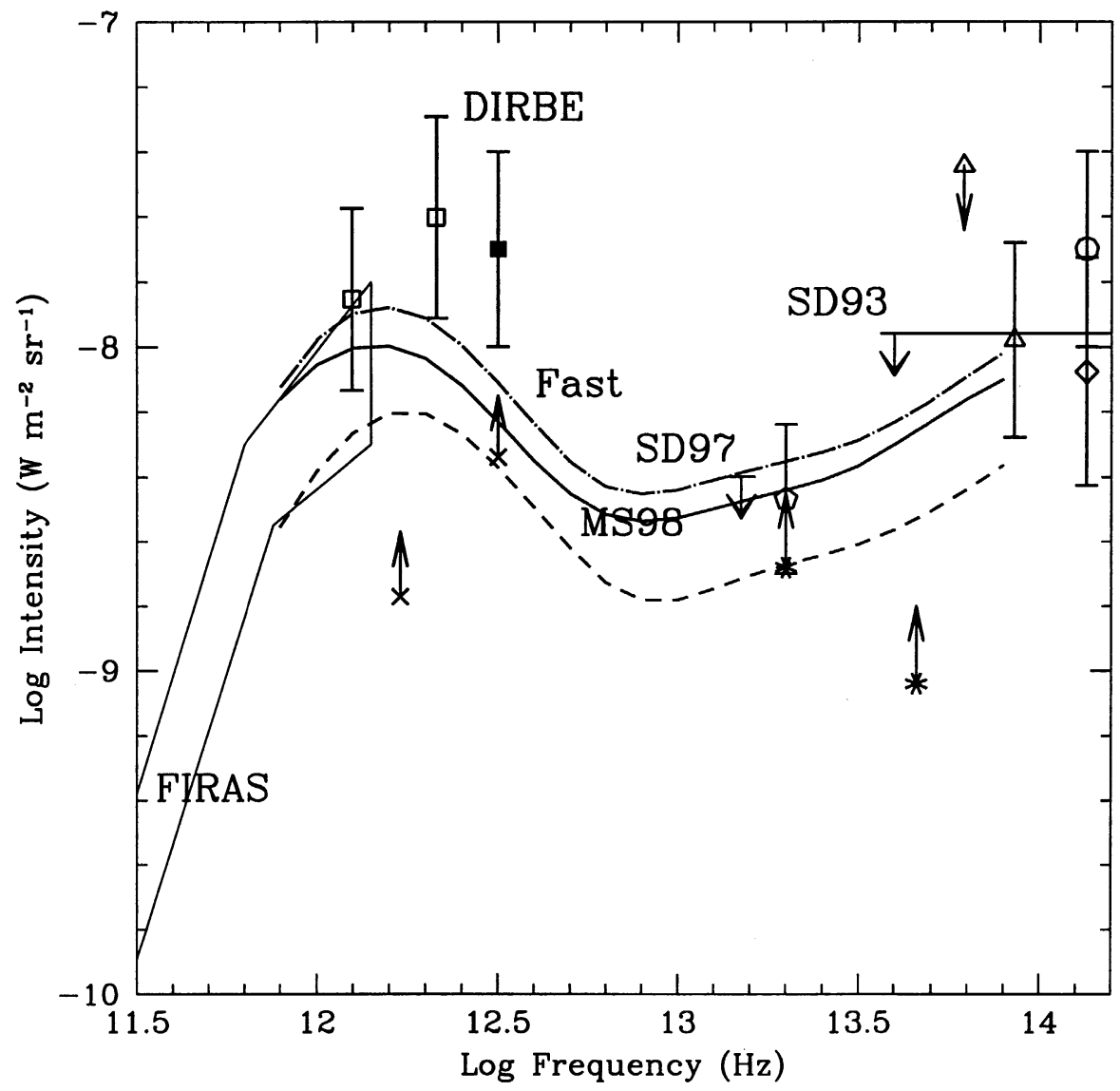

Figure 1. The spectral energy distribution (SED) of the extragalactic infrared radiation calculated by Malkan \& Stecker $(1998,2000)$. The dashed line (lower IIRF curve) and the solid line (middle IIRF curve) correspond to the middle and upper curves calculated by Malkan \& Stecker (1998) with redshift-evolution assumptions as described in the text. The dot-dashed line (Malkan \& Stecker 2000) corresponds to a "fast evolution" case with $Q=4.1$ and $z_{\text {flat }}=1.3$. Representative data are also shown with $2 \sigma$ error bars as follows. From left to right, FIRAS polygon: Fixsen et al. 1998; open squares: Hauser et al. (1998); solid square: Lagache et al. (2000); pentagon: Altieri et al. (1999); open triangles: Dwek and Arendt (1998); open circle: Gorjian, Wright \& Chary (2000); diamond: Totani, (2000) (see also Pozzetti et al. 2000). The lower limits with crosses are from ISOPHOT (Puget et al. 1999) and the lower limits with asterisks are from ISOCAM (Elbaz et al. 1999). The upper limits are from Stecker \& de Jager (1993)(SD93) and Stecker \& de Jager (1997)(SD97). 


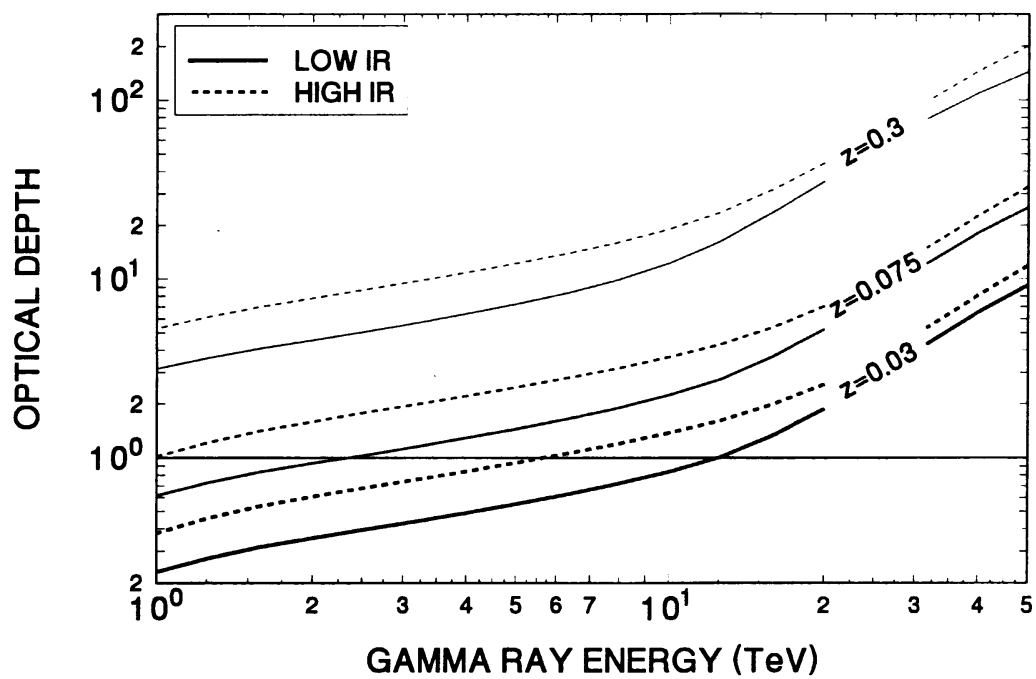

Figure 2. Optical depth versus energy for $\gamma$-rays originating at various redshifts obtained using the SEDs corresponding to the lower IIRF (solid lines) and middle IIRF (dashed lines) levels shown in Fig. 1 (from SD98). Note that the line styles in this Figure are the reverse of those in Figure 1.

between the cold dust peak in the far-infrared and cool star peak in the near IR is filled in more in the MS98 results and is not as pronounced as in previously derived models of the IR background SED. As can be seen in Figure 1, the background SED predicted in MS98 is in excellent agreement with the results obtained from ultradeep ISOCAM galaxy count observations at $15 \mu \mathrm{m}$ (Altieri et al. 1999).

The SD98 calculations predict that intergalactic absorption should only slightly steepen the spectra of Mrk 421 and Mrk 501 below $\sim 7 \mathrm{TeV}$, which is consistent with the data in the published literature. The SD98 calculations further predict that intergalactic absorption should turn over the spectra of these sources at energies greater than $\sim 15 \mathrm{TeV}$.

\section{The Multi-TeV Spectrum of Mrk 501 and its Interpretation}

The HEGRA group has observed the BL Lac object Mrk 501 in the flaring phase, obtaining an energy spectrum for this source up to an energy of $24 \mathrm{TeV}$ (Aharonian et al. 1999). The Mrk 501 spectrum obtained by the HEGRA group is well fitted by a source spectrum power-law of spectral index $\sim 2$ steepened in the multi-TeV energy range by intergalactic absorption with the optical depth calculated by SD98 (Konopelko, et al. 1999). Figure 3 clearly shows this. 


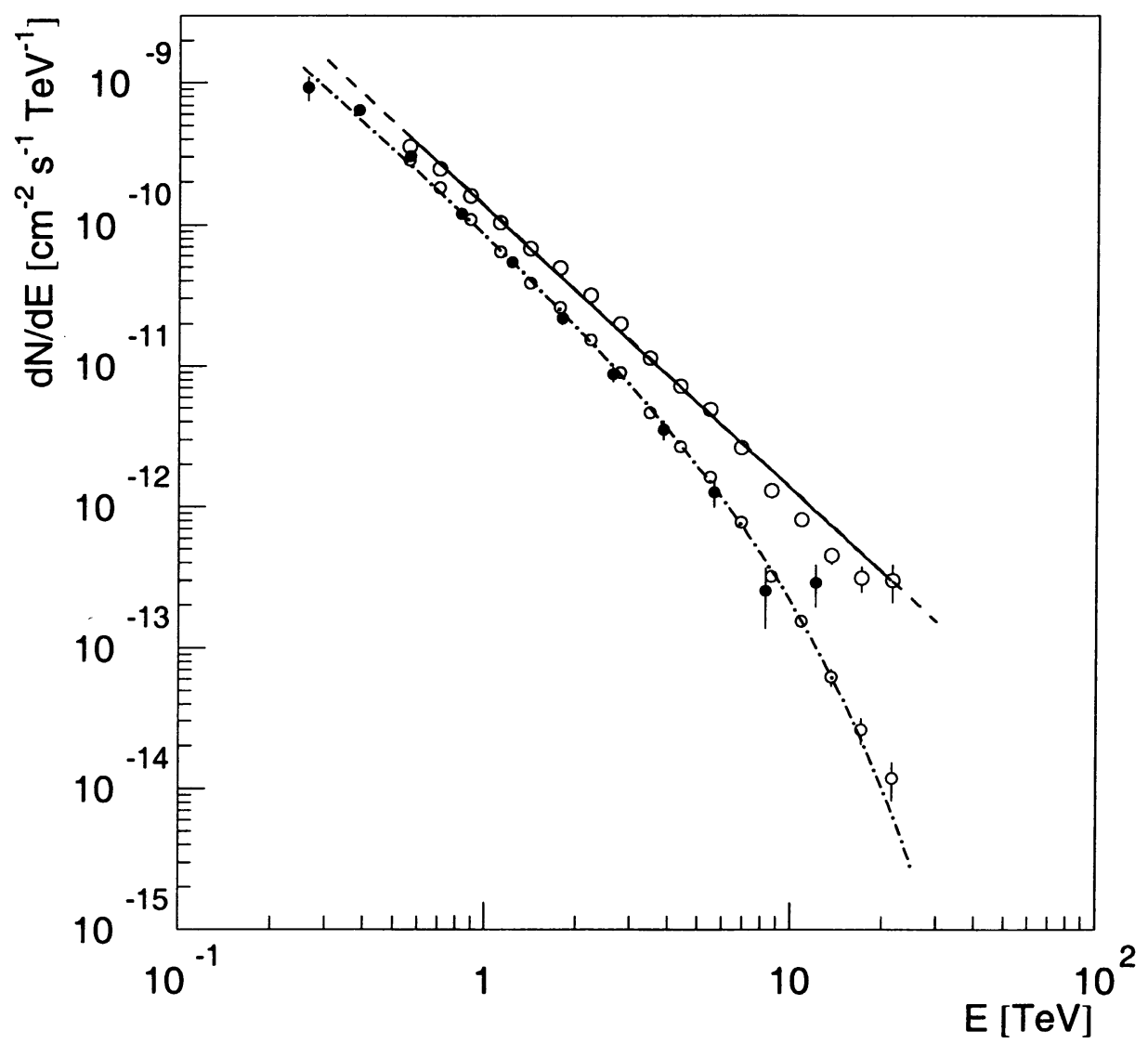

Figure 3. The HEGRA and Whipple telescope data on Mrk 501 in the flaring state is indicated by the lower line. The upper line and points show the intrinsic spectrum of the source with the effect of extragalactic absorption removed. The absorption is calculated using the middle line IIRF spectrum shown in Figure 1. See also Figure 2. (From Konopelko et al. 1999.) 


\section{Reconciling the Infrared and Gamma-Ray Observations}

Malkan \& Stecker (2000) have used their empirically based model (MS98) to predict infrared luminosity functions and deep infrared galaxy counts at various wavelengths. They have also examined their predictions for the IIRF for comparison with the subsequent determinations from the COBE-DIRBE data analysis. Using the formalism of luminosity evolution proportional to $(1+z)^{Q}$ out to a redshift of $z_{\text {flat }}$ and constant (no evolution) for $z_{\text {flat }}<z<z_{\max }=4$, they find that a comparison of their predictions with current ISO galaxy counts at 15 and $175 \mu \mathrm{m}$ favor their "Baseline Model" with $Q=3.1$ and $z_{\text {flat }}=2$ (the middle curve in Figure 1). The $\gamma$-ray limits (SD97) also favor $Q \sim 3$.

On the other hand, the COBE-DIRBE far infrared determinations seem to favor a stronger evolution with $Q>4$ up to $z_{\text {flat }}=1$. For example, the upper curve in Figure 1 (Malkan \& Stecker 2000) assumes $Q=4.1$ and $z_{\text {flat }}=1.3$. This curve gives a flux in the mid-infrared which is a factor of $\sim 1.8$ above the "Baseline Model" and would imply a corresponding increase in the opacity of the universe to multi-TeV $\gamma$-rays. (Thus, one should multiply the appropriate bracketed numbers in Table 1 by a factor of 1.8.) Therefore, $\tau(15 \mathrm{TeV})$ would increase from $\sim 2$ to $\sim 4$ in the case of Mrk 501 and Mrk $421(z \simeq 0.03)$.

This prima facie conflict can be resolved in two ways: either (a) the COBEDIRBE far-infrared estimates may suffer from under subtraction of foreground emission and therefore are too high, or (b) the ISOPHOT galaxy counts may be missing a significant fraction of sources. In this latter case, one may also have to require that the $\gamma$-ray results are wrong in that the HEGRA energy determinations have been overestimated, mimicking the effect of absorption which would be produced by a lower IIRF. Another possibility is one involving new physics, viz. that Lorentz invariance may be broken, allowing the universe to be transparent to multi-TeV photons (Coleman \& Glashow 1999; Kifune 1999). This "new physics" scenario presents problems in that the Mrk 501 spectrum does exhibit exactly the characteristics expected for high-energy $\gamma$-ray absorption from pair-production (Konopelko et al. 1999). However, we will discuss this possibility more quantitatively in the next Section (Glashow \& Stecker 2000).

Possibility (a) finds support in the independent analysis of the COBE data by Lagache et al. (1999) who obtain a flux at $140 \mu \mathrm{m}$ which is only $60 \%$ of the flux obtained by Hauser et al. (1998) shown in Figure 1. Lagache et al. (2000) also obtained a smaller flux at $240 \mu \mathrm{m}$. In this regard, one should also note that the results reported by Hauser et al. (1998) were at the $4 \sigma$ level.

If the far-infrared galaxy counts are incomplete (possibility (b)), this would imply stronger evolution in the far-infrared emission of galaxies than in the midinfrared. Although the MS98 model already includes some differential evolution of this type, based on the data of Spinoglio et al. (1995), it is conceivable that starburst galaxies at redshifts $\sim 1$ might produce an even higher ratio of $\sim 60 \mu \mathrm{m}$ to $\sim 7 \mu \mathrm{m}$ rest-frame fluxes than their present-day counterparts. Radiation at these widely separated wavelengths is known to be emitted by quite different dust grains which could have different evolutionary development, particularly for ULIRGs (ultraluminous infrared galaxies) and AGN (active galactic nuclei). One should note that this possibility can make the $\gamma$-ray and infrared data compatible, since absorption of $\sim 15 \mathrm{TeV} \gamma$-rays is caused by interactions with mid-infrared $(\sim 20 \mu \mathrm{m})$ photons and not $140 \mu \mathrm{m}$ far-infrared photons. 


\section{Breaking Lorentz Invariance}

With the idea of spontaneous symmetry breaking in particle physics came the suggestion that Lorentz invariance (LI) might be weakly broken at high energies (Sato \& Tati 1972). Although no real quantum theory of gravity exists, it was suggested that LI might be broken as a consequence of such a theory (Amelino-Camilia et al. 1998). A simpler formulation for breaking LI by a small first order perturbation in the electromagnetic Lagrangian which leads to a renormalizable treatment has been given by Coleman \& Glashow (1999). The breaking of LI at high energies is one way of avoiding the predicted, but unseen, "GZK" cutoff in the ultrahigh energy cosmic-ray spectrum owing to photomeson interactions with $2.7 \mathrm{~K}$ cosmic background photons (Greisen 1966; Zatsepin \& Kuz'min 1966), which produces an effective absorption mean-free-path for ultrahigh energy cosmic rays in intergalactic space of $<100 \mathrm{Mpc}$ (Stecker 1968).

It has recently been suggested that LI breaking could also make the universe transparent to high energy $\gamma$-rays (Kifune 1999). Such LI breaking implies a preferred frame of reference in the universe which would naturally be associated with a rest frame for the $2.7 \mathrm{~K}$ cosmic background radiation. We follow here the formalism proposed by Coleman \& Glashow (1999) for LI breaking. Within this scenario, the maximum attainable velocity of an electron, $c_{e} \neq c_{\gamma}$, the velocity of the photon. Let us consider the case where $c_{e}>c_{\gamma}$ and we define

$$
c_{e} \equiv c_{\gamma}(1+\delta), \quad \delta \ll 1
$$

In this case, electrons above an energy $E_{\max }=\gamma_{\max } m_{e}$ will be superluminal. They will radiate Cherenkov light if their velocity is large enough, i.e., if $\beta>\left(c_{\gamma} / c_{e}\right)$, which implies that $\gamma_{e}^{2}>\left[1-\left(c_{\gamma} / c_{e}\right)^{2}\right]^{-1}$. This determines the maximum electron energy above which electrons would rapidly lose energy by Cherenkov radiation:

$$
E_{m a x}=m_{e}(2 \delta)^{-1 / 2}
$$

(In this Section, we adopt the standard particle physics convention $c=1$.) Since electrons are seen in the cosmic radiation up to an energy $\sim 1 \mathrm{TeV}$, this implies that $E_{\max }>1 \mathrm{TeV}$, which gives an upper limit on $\delta$ of $1.3 \times 10^{-13}$.

If $L I$ is broken so that $\delta>0$, the threshold energy for the pair production process is altered because the square of the four-momentum becomes

$$
2 \epsilon E_{\gamma}(1-\cos \theta)-2 E_{\gamma}^{2} \delta=4 \gamma^{2} m_{e}^{2}>4 m_{e}^{2}
$$

where $\epsilon$ is the energy of the low energy (infrared) photon and $\theta$ is the angle between the two photons. The second term on the left-hand-side comes from the fact that $c_{\gamma}=\partial E_{\gamma} / \partial p_{\gamma}$.

For head-on collisions $(\cos \theta=-1)$ the minimum low energy photon energy for pair production becomes

$$
\epsilon_{\min }=m_{e}^{2} / E_{\gamma}+\left(E_{\gamma} \delta\right) / 2
$$

It follows that the condition for a significant increase in the energy threshold for pair production is 


$$
\left(E_{\gamma} \delta\right) / 2 \geq m_{e}^{2} / E_{\gamma}
$$

or, equivalently, $\delta$ must be greater than $2 m_{e}^{2} / E_{\gamma}^{2}$.

Thus, for a significant decrease in the optical depth to Mrk 501 for $E_{\gamma}=15$ $\mathrm{TeV}$, we must have $\delta \geq 2.4 \times 10^{-15}$.

The effect of breaking LI is to exclude photons of energy below $\epsilon_{\min }$ from pair producing, thus reducing the number of target photons. In this way, one can exclude the high flux of far-infrared photons implied by the COBE-DIRBE analysis from participating in the absorption process while still allowing midinfrared photons to produce an absorption feature.

One can rule out this hypothesis if one observes electrons with energies much greater than $1 \mathrm{TeV}$. This would reduce the upper limit on $\delta$ to the point where there will be no significant reduction in absorption by pair-production interactions with infrared photons.

On the other hand, if one observes $\gamma$-rays above $100 \mathrm{TeV}$ from an extragalactic source, this would be strong evidence for LI breaking. This is because the very large density $\left(\sim 400 \mathrm{~cm}^{-3}\right)$ of $3 \mathrm{~K}$ cosmic microwave photons would otherwise absorb $>100 \mathrm{TeV} \gamma$-rays within a distance of $\sim 10 \mathrm{kpc}$ (see eq. (1)).

Finally, we note that even if absorption is reduced in Mrk 501, one can still have absorption in sources at higher redshifts owing to interactions with higher energy photons (see next Section).

\section{Absorption of Gamma-Rays at High Redshifts}

We now discuss the absorption of 10 to $500 \mathrm{GeV} \gamma$-rays at high redshifts. In order to calculate such high-redshift absorption properly, it is necessary to determine the spectral distribution of the intergalactic low energy photon background radiation as a function of redshift as realistically as possible out to frequencies beyond the Lyman limit. This calculation, in turn, requires observationally based information on the evolution of the spectral energy distributions (SEDs) of IR through UV starlight from galaxies, particularly at high redshifts.

Conversely, observations of high-energy cutoffs in the $\gamma$-ray spectra of blazars as a function of redshift, which may enable one to separate out intergalactic absorption from redshift-independent cutoff effects, could add to our knowledge of galaxy formation and early galaxy evolution. In this regard, it should be noted that the study of blazar spectra in the 10 to $300 \mathrm{GeV}$ range is one of the primary goals of a next generation space-based $\gamma$-ray telescope GLAST (Gammaray Large Area Space Telescope) (Bloom 1996) as well as VERITAS and other future ground based $\gamma$-ray telescopes.

Salamon and Stecker (1998) (hereafter SS98) have calculated the $\gamma$-ray opacity as a function of both energy and redshift for redshifts as high as 3 by taking account of the evolution of both the SED and emissivity of galaxies with redshift. In order to accomplish this, they adopted the recent analysis of Fall et al. (1996) and also included the effects of metallicity evolution on galactic SEDs. Their results indicate that the extragalactic $\gamma$-ray background spectrum from blazars should steepen significantly above $20 \mathrm{GeV}$, owing to extragalactic absorption. 
Future observations of such a steepening would thus provide a test of the blazar origin hypothesis for the $\gamma$-ray background radiation.

\subsection{Redshift Dependence of the Intergalactic Low Energy SED}

Pei and Fall (1995) have devised a method for calculating stellar emissivity which bypasses the uncertainties associated with estimates of poorly defined luminosity distributions of evolving galaxies. The core idea of their approach is to relate the star formation rate directly to the evolution of the neutral gas density in damped Lyman- $\alpha$ systems, and then to use stellar population synthesis models to estimate the mean co-moving stellar emissivity $\mathcal{E}_{\nu}(z)$ of the universe as a function of frequency $\nu$ and redshift $z$. The SS98 calculation of stellar emissivity closely follows this elegant analysis, with minor modifications.

Damped Lyman- $\alpha$ systems are high-redshift clouds of gas whose neutral hydrogen surface density is large enough $\left(>2 \times 10^{20} \mathrm{~cm}^{-2}\right)$ to generate saturated Lyman- $\alpha$ absorption lines in the spectra of background quasars that happen to lie along and behind common lines of sight to these clouds. These gas systems are believed to be either precursors to galaxies or young galaxies themselves, since their neutral hydrogen (HI) surface densities are comparable to those of spiral galaxies today, and their co-moving number densities are consistent with those of present-day galaxies (Worthy 1986; Pei \& Fall 1995). It is in these systems that initial star formation presumably took place, so there is a relationship between the mass content of stars and of gas in these clouds; if there is no infall or outflow of gas in these systems, the systems are "closed", so that the formation of stars must be accompanied by a reduction in the neutral gas content. Such a variation in the HI surface densities of Lyman- $\alpha$ systems with redshift is seen, and is used by Pei and Fall (1995) to estimate the mean cosmological rate of star formation back to redshifts as large as $z=5$.

Following Fall, Charlot \& Pei (1996), SS98 used the Bruzual-Charlot model (Charlot \& Bruzual 1991; Bruzual \& Charlot 1993) corresponding to a Salpeter initial mass function, $\phi(M) d M \propto M^{-2.35} d M$, where $0.1 M_{\odot}<M<125 M_{\odot}$. The mean co-moving emissivity $\mathcal{E}_{\nu}(t)$ was then obtained by convolving over time $t$ the specific luminosity with the mean co-moving mass rate of star formation. SS98 also obtained metallicity correction factors for stellar radiation at various wavelengths. Increased metallicity gives a redder population spectrum (Worthy 1994; Bertelli, et al. 1994).

SS98 calculated stellar emissivity as a function of redshift at $0.28 \mu \mathrm{m}$, $0.44 \mu \mathrm{m}$, and $1.00 \mu \mathrm{m}$, both with and without a metallicity correction. Their results agree well with the emissivity obtained by the Canada-French Redshift Survey (Lilly, et al 1996) over the redshift range of the observations $(z \leq 1)$.

The stellar emissivity in the universe is found to peak at $1 \leq z \leq 2$, dropping off steeply at lower redshifts and more slowly at higher redshifts. Indeed, Madau, Pozzetti \& Dickinson (1998) have used observational data from the Hubble Deep Field to show that metal production has a similar redshift distribution, such production being a direct measure of the star formation rate.

The co-moving radiation energy density $u_{\nu}(z)$ is the time integral of the co-moving emissivity $\mathcal{E}_{\nu}(z)$,

$$
u_{\nu}(z)=\int_{z}^{z_{\max }} d z^{\prime} \mathcal{E}_{\nu^{\prime}}\left(z^{\prime}\right) \frac{d t}{d z}\left(z^{\prime}\right) e^{-\tau_{\mathrm{eff}}\left(\nu, z, z^{\prime}\right)},
$$


where $\nu^{\prime}=\nu\left(1+z^{\prime}\right) /(1+z)$ and $z_{\max }$ is the redshift corresponding to initial galaxy formation. The extinction term $e^{-\tau_{\text {eff }}}$ accounts for the absorption of ionizing photons by the clumpy intergalactic medium (IGM) that lies between the source and observer. Although the IGM is effectively transparent to non-ionizing photons, the absorption of photons by HI, HeI and HeII can be considerable (Madau 1995).

\subsection{The Gamma-Ray Opacity at High Redshifts}

SS98 evaluated the co-moving energy density $u_{\nu}(z)$ using equation (9) above. They then calculated the optical depth for $\gamma$-rays owing to electron-positron pair production interactions with photons of the stellar radiation background. This can be determined from the expression (Stecker, De Jager \& Salamon 1992)

$$
\tau\left(E_{0}, z_{e}\right)=c \int_{0}^{z_{e}} d z \frac{d t}{d z} \int_{0}^{2} d x \frac{x}{2} \int_{0}^{\infty} d \nu(1+z)^{3}\left[\frac{u_{\nu}(z)}{h \nu}\right] \sigma_{\gamma \gamma}(s)
$$

where $s=2 E_{0} h \nu x(1+z), E_{0}$ is the observed $\gamma$-ray energy at redshift zero, $\nu$ is the frequency at redshift $z, z_{e}$ is the redshift of the $\gamma$-ray source, $x=(1-\cos \theta), \theta$ being the angle between the $\gamma$-ray and the soft background photon, $h$ is Planck's constant $(\epsilon=h \nu)$, and the pair production cross section $\sigma_{\gamma \gamma}$ is zero for centerof-mass energy $\sqrt{s}<2 m_{e} c^{2}, m_{e}$ being the electron mass. Above this threshold,

$$
\sigma_{\gamma \gamma}(s)=\frac{3}{16} \sigma_{\mathrm{T}}\left(1-\beta^{2}\right)\left[2 \beta\left(\beta^{2}-2\right)+\left(3-\beta^{4}\right) \ln \left(\frac{1+\beta}{1-\beta}\right)\right],
$$

where $\beta=\left(1-4 m_{e}^{2} c^{4} / s\right)^{1 / 2}$.

Figure 4, based on the results of SS98, shows the predicted critical energy for a 1/e absorption by pair production versus redshift, obtained for the cases of correction for metallicity evolution and no correction. Absorption of $\gamma$-rays of energies below $\sim 15 \mathrm{GeV}$ is negligible.

The weak redshift dependence of the opacity at the higher redshifts, as shown in Figure 4, indicates that the opacity is not very sensitive to the initial epoch of galaxy formation, contrary to the speculation of MacMinn and Primack (1996). In fact, the uncertainty in the metallicity correction (see Figure 4) would obscure any dependence on $z_{\max }$ even further.

\subsection{The Effect of Absorption on the Spectra of Blazars and the Gamma-Ray Background}

With the $\gamma$-ray opacity $\tau\left(E_{0}, z\right)$ calculated out to $z=3$, the cutoffs in blazar $\gamma$-ray spectra caused by extragalactic pair production interactions with stellar photons can be predicted. The left graph in Figure 5, from SS98, shows the effect of the intergalactic radiation background on a few of the blazars observed by EGRET, viz., $1633+382,3 \mathrm{C} 279,3 \mathrm{C} 273$, and Mrk 421, assuming that the mean spectral indices obtained for these sources by EGRET extrapolate out to higher energies attenuated only by intergalactic absorption. (Observed cutoffs in blazar spectra may be intrinsic cutoffs in $\gamma$-ray production in the source, or may be caused by intrinsic $\gamma$-ray absorption within the source itself.) 


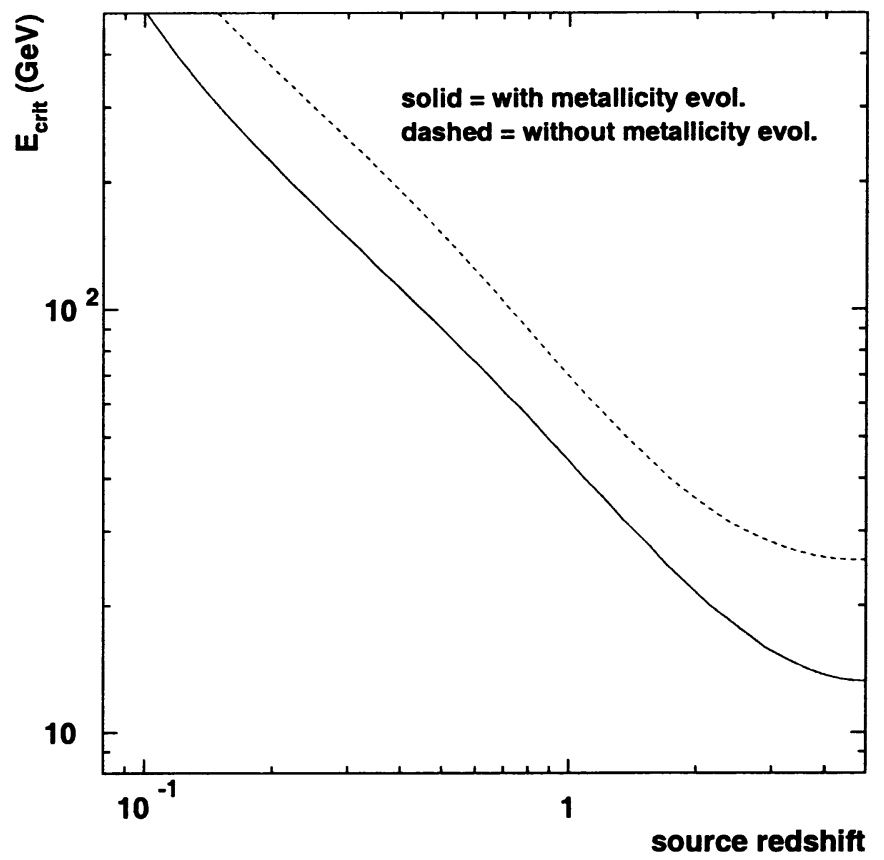

Figure 4. The critical energy for $\gamma$-ray absorption above which the optical depth is predicted to be greater than 1 as a function of the redshift of the source (obtained from the results of SS98).

The right hand graph in Figure 5 shows the background spectrum predicted from unresolved blazars (Stecker \& Salamon 1996; Salamon \& Stecker 1998) compared with the EGRET data (Sreekumar, et al. 1998). Note that the predicted spectrum steepens above $20 \mathrm{GeV}$, owing to extragalactic absorption by pair-production interactions with intergalactic optical and infrared photons, particularly at high redshifts.

\section{Conclusions}

Studies of the absorption of high energy $\gamma$-rays in the spectra of extragalactic objects can be used to probe both the present intergalactic infrared background and the infrared and optical radiation that existed in intergalactic space at higher redshifts. The results of such observations are free of the effects of solar system and galactic foreground contamination. Such studies are most effective when combined with direct infrared observations in a synoptic approach.

High energy $\gamma$-ray observations have already provided the best constraints on the mid-infrared background and they support a galaxy evolution scenario where emissivity evolution in the mid-infrared evolved as $\sim(1+z)^{Q}$ with $Q \sim$ 3. This is consistent with observations of galaxy counts using ISOCAM. On the other hand, the analysis of COBE--DIRBE observations favors a stronger evolution in far-infrared emissivity with $Q>4$. 

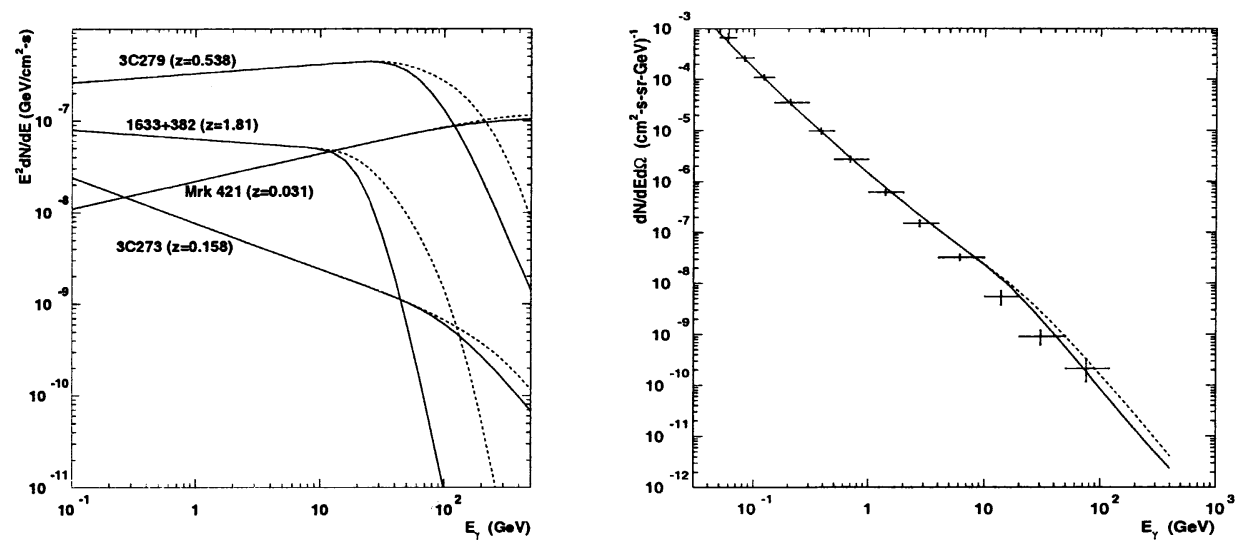

Figure 5. The left graph shows the effect of intergalactic absorption by pair-production on the power-law spectra of four prominent blazars: $1633+382(z=1.81), 3 \mathrm{C} 279(z=0.54), 3 \mathrm{C} 273(z=0.15)$, and Mrk $421(z=0.031)$; The right graph shows the extragalactic $\gamma$-ray background spectrum predicted by the unresolved blazar model of Stecker \& Salamon (1996) with absorption included, calculated for a mean EGRET point-source sensitivity of $10^{-7} \mathrm{~cm}^{-2} \mathrm{~s}^{-1}$, compared with the EGRET data on the $\gamma$-ray background (Sreekumar et al. 1998). The solid (dashed) curves are calculated with (without) the metallicity correction function (from SS98).

There are various possible interpretations of these results; among them is the possibility that special relativity is modified at high energies. Future $\gamma$-ray and infrared observations will be needed to resolve this situation.

Acknowledgments. I would like to acknowledge my collaborators in much of this work. They are (in alphabetical order) Okkie De Jager, Sheldon Glashow, Matthew Malkan and Michael Salamon.

\section{References}

Aharonian, F., et al. 1997, A\&A, 327, L5

Aharonian, F., et al. 1999, A\&A, 342, 69

Altieri, B., et al. 1999, A\&A, 343, L65

Amelino-Camilia, G., et al. 1998, Nature, 393, 763

Bertelli, G., et al. 1994, A\&AS, 106, 275.

Bertin, E., Dennefeld, M., \& Moshir M. 1997, A\&A, 323, 685

Biller, S.D. et al. 1998, Phys. Rev. Lett., 80, 2992

Blain, A.W., \& Natarajan, P. 2000, MNRAS, 312, L39

Bloom, E.D. 1996, Space Sci.Rev., 75, 109

Bruzual, A.G., \& Charlot, S. 1993, ApJ, 457, 538 
Charlot, S., \& Bruzual A.G. 1991, ApJ, 405, 625

Coleman, S., \& Glashow, S.L. 1999, Phys.Rev.D, 591, 528 (id. 116008)

Dwek, E., \& Arendt, R. 1998, ApJ, 508, L9

Dwek, E., \& Slavin, J. 1994, ApJ, 436, 696

Elbaz, D., et al. 2000, A\&A Letters, in press, e-print astro-ph/9910496

Fall, S. M., Charlot, S., \& Pei, Y.C. 1996 ApJ, 464, L43

Fixsen, D. J., et al. 1998, ApJ, 508, 123

Franceschini, A., et al. 1998, MNRAS, 296, 709

Glashow, S.L., \& Stecker, F.W. 2000, in preparation

Gorjian, V., Wright, E. L. \& Chary, R. R. 2000, ApJ, 536, 550

Gratton, R. G. et al. 1997, PASP, 402, 651

Greisen, K. 1966, Phys.Rev.Lett, 16, 148

Hauser, M., et al. 1998, ApJ, 508, 25

Hopkins, A.M., Connolly, A. J. \& Szalay, A. S. 2000, AJ, in press, e-print astro$\mathrm{ph} / 0009073$

Kifune, T. 1999 ApJ, 518, L21

Konopelko, A.K. Kirk, J.G., Stecker, F.W., \& Mastichiadas, A. 1999, ApJ, 518, L13

Lagache, G., Haffner, L. M., Reynolds, R. J., \& Tufte, S. L. 2000, A\&A, 354, 247

Lawrence, A., et al. 1986, MNRAS, 219, 687

Lilly, S.J., et al. 1996, ApJ, 460, L1

MacMinn, D., \& Primack, J. R. 1996, Space Sci.Rev., 75, 413

Madau, P. 1995, ApJ, 441, 18

Madau, P. Pozzetti, L., \& Dickinson, M. 1998, ApJ, 498, 106

Malkan, M. A., \& Stecker, F. W. 1998, ApJ, 496, 13 (MS98)

Malkan, M. A., \& Stecker, F. W. 2000, ApJ, submitted, astro-ph/0009500

McEnery, J. E., et al. 1997, Proc. 25th Intl. Cosmic Ray Conf., Durban, S. A., 3,257

Pei, Y.C., \& Fall, S.M. 1995, ApJ, 454, 69

Pozzetti, L., et al. 2001, in The Extragalactic Background and its Cosmological Implications, IAU Symp. Vol. 204, ed. M. Harwit and M. G. Hauser, in press

Puget, J-L., et al. 1999, A\&A, 354, 29

Salamon, M. H., \& Stecker, F. W. 1998, ApJ, 493, 547 (SS98)

Sato, H., \& Tati, T. 1972, Prog. Theor. Phys., 47, 1788

Sreekumar, P., et al. 1998, ApJ, 494, 523

Spinoglio, L., et al. 1995, ApJ, 453, 616

Stanev, T., \& Franceschini, A. 1998, ApJ, 494, 159

Stecker, F. W. 1968, Phys.Rev.Lett, 21, 1016

Stecker, F. W. \& De Jager, O. C. 1993, ApJ, 415, L71 
Stecker, F. W., \& De Jager, O. C. 1997, in Towards a Major Atmospheric Cerenkov Detector V, Proc. Kruger National Park Workshop on TeV Gamma Ray Astrophysics, ed. O.C. De Jager (Potchefstroom: Wesprint), 39

Stecker, F. W., \& De Jager, O. C. 1998, A\&A, 334, L85 (SD98)

Stecker, F. W., De Jager, O.C., \& Salamon, M. H. 1992, ApJ, 390, L49

Stecker, F. W., De Jager, O. C., \& Salamon, M. H. 1996, ApJ, 473, L75

Stecker, F. W., \& Salamon, M. H. 1996, ApJ, 464, 600

Steidel, C. C., et al. 1999, ApJ, 519, 1

Totani, T., et al. 2001, in The Extragalactic Background and its Cosmological Implications, IAU Symp. Vol. 204, ed. M. Harwit and M. G. Hauser, in press

Worthey, G. 1994, ApJS, 95, 107.

$\mathrm{Xu}, \mathrm{C}$., et al. 1998, ApJ, 508, 576

Zatsepin, G. T., \& Kuz'min, V.A. 1966, JETP Lett., 4, 78

\section{Discussion}

Alberto Carramiñana: Two questions:

1. Do GeV observations set limits on the UV background?

2. Is absorption in the source a problem, given the local radiation environment?

Floyd Stecker:

1. According to our calculations (Salamon \& Stecker, 1998, see reference above), blazars even at high redshifts will have absorption rollovers at $E_{\gamma} \geq$ $20 \mathrm{GeV}$. The EGRET results for individual blazars do not extend above $10 \mathrm{GeV}$ because EGRET's sensitivity was not good enough to get photon counts above $10 \mathrm{GeV}$. Hopefully, the next generation GLAST telescope will get spectra extending to much higher energies.

2. Absorption in Mrk 501 should not be a problem because this is a giant elliptical galaxy with little dust and therefore little $\sim 20 \mu \mathrm{m}$ emission.

Jim Felten: Perhaps this is related to the previous question. Now that there are many data on the IR background, you should use the observed spectrum, rather than your semi-empirical model, to calculate the optical depths. In your last figure I believe you did this. But did I hear you take issue with something in the observed spectrum which Hauser showed earlier in the day? Please expand on this. Are there important gaps in the spectrum? Can you add some significant limits?

Stecker: There are no data in the mid-IR from COBE because of large zodiacal light contamination. Our calculations are in excellent agreement with the $3-5 \mu \mathrm{m}$ results of Dwek \& Arendt. The $\gamma$-ray limit of $\sim 4 \mathrm{nW} \mathrm{m}^{-2} \mathrm{sr}^{-1}$ at $\sim 20 \mu \mathrm{m}$ (Stecker \& De Jager, 1977, see reference above) gives the best upper limit on the mid-IR flux. 